\title{
Changes in Doppler indices throughout pregnancy in queens
}

\author{
Melih Uçmak, Ozge Turna, Gamze Evkuran Dal, Zeynep Günay Uçmak, \\ Mehmet Can Gündüz
}

\begin{abstract}
${ }^{1}$ Department of Obstetrics and Gynecology, Faculty of Veterinary Medicine, Istanbul University-Cerrapaşa, Istanbul, Turkey
\end{abstract}

Received April 25, 2019

Accepted January 28, 2020

\begin{abstract}
Doppler ultrasonography (D-USG) is a non-invasive technique that has become an increasingly essential tool in veterinary theriogenology. The aim of this study was to evaluate the changes of Doppler indices in the foetal aorta (FA), foetal vena cava (FVC), umbilical artery (UA), and uteroplacental artery (UPA) in pregnant queens, thereby advancing knowledge about Doppler findings in normal feline pregnancy. The study was conducted with 10 pregnant queens with known gestational age. Doppler ultrasonography was performed every three days beginning from the $21^{\text {st }}$ day to the $60^{\text {th }}$ day of pregnancy. The pulsatility index (PI) and resistance index (RI) of FA, FVC, UA and UPA were recorded during the D-USG examinations. Although a declining trend was seen in the mean RI of FA, a comparison with earlier sampling periods revealed that significant decreases $(P<0.05)$ did not occur until Days 30 and 60 . While PI of FVC was relatively constant, RI varied througout pregnancy. The PI and RI of UPA values were significantly lower $(P<0.05)$ on Day 60 when compared to the first five sampling periods for PI and the first six sampling periods for RI. The study concludes that the decrease in UA indices is a precursor for delivery time in queens. However, there is a need for further comparative studies involving impaired pregnancies.
\end{abstract}

Doppler ultrasonography, pulsatility index, resistance index, pregnant queen

Doppler ultrasonography (D-USG) is a non-invasive technique that provides information about maternal and foetal haemodynamics in pregnancy (Fleischer et al. 1994; Erdogan 2018). It is a widely used technique for the evaluation of foeto-maternal circulation in pregnancy. The use of D-USG in perinatology encompasses two techniques: uteroplacental Doppler, and foetal arterial-venous Doppler (Lees et al. 2003). Blood vessels can be evaluated qualitatively via waveforms, and semi-quantitatively via Doppler indices. Because the Doppler indices are calculated as a ratio, they are virtually angle-independent (Maulik et al. 2011).

The use of D-USG in veterinary practice has increased over the last decade. The haemodynamic characteristics of maternal and foetal vessels throughout pregnancy were previously reported in mares (Ouset et al. 2012; Ferreira et al. 2015), cows (Kelley et al. 2017), ewes (Elmetwally et al. 2016; Turna Yilmaz et al. 2017), goats (Serin et al. 2010), rabbits (Polisca et al. 2010), bitches (Di Salvo et al. 2006), and queens (Scotti et al. 2008; Blanco et al. 2014; Blanco et al. 2016). As these studies show, the evaluation of the haemodynamic characteristics of maternal and foetal vessels by Doppler ultrasonography in pregnancy provides early diagnosis of foetal hypoxia, allows for appropriate treatment protocols to be determined, and leads to the successful management of pregnancy (Filkins and Koos 2005).

Part of the increasing interest in the haemodynamic characteristics of different species in veterinary obstetrics, Doppler evaluation of maternal and foetal vessels in normal feline pregnancy was first described in 2008 by Scotti et al. Doppler findings of impaired pregnancies were first reported by Turna et al. (2014), based on findings

Address for correspondence:

Assoc. Prof. Ozge Turna

Department of Obstetrics and Gynecology

Faculty of Veterinary Medicine, Istanbul University-Cerrahpasa

Avcilar 34320, Istanbul, Turkey

Tel: $+902124737070 / 17315$

E-mail: turnaozge@hotmail.com; turnao@istanbul.edu.tr http://actavet.vfu.cz/ 
from the pregnancies of four cats. It is important to compare the formation of speciesspecific intrauterine growth curves in healthy pregnancies with those that occur in impaired pregnancies (Erdogan 2018). The aim of this study is to advance knowledge about Doppler findings for normal feline pregnancy.

\begin{abstract}
Materials and Methods
Study design

This study was conducted at the Department of Obstetrics and Gynecology, Faculty of Veterinary Medicine, at Istanbul University-Cerrahpaşa. Crossbred, clinically healthy queens $(\mathrm{n}=10)$ and tomcats $(\mathrm{n}=2), 3-6$ years of age and weighing an average of $3.4 \mathrm{~kg}$, were used in this study. The cats were kept in individual cages allowing exposure to natural photoperiods and regional springtime weather. They were also allowed to roam in an outdoor area at certain times of the day. They were fed a commercially available dry food and received water ad libitum.

The queens were observed for oestrus signs once a day and presented to a tomcat in case of oestrus detection. The day of mating was defined as Day 0. Pregnancy was confirmed with B-mode real-time ultrasonography (MyLab 5-Vet ESAOTE ${ }^{\circledR}$, Genova, Italy) using a $5 \mathrm{MHz}$ microconvex probe on the $15^{\text {th }}$ day after mating. The foetal thoracic aorta (FA), foetal vena cava (FVC), umbilical artery (UA) and uteroplacental artery (UPA) of the most caudal foetuses were individually visualized (12) using colour flow mapping. The FA was easily observed throughout its arch. The display area of FVC was near the right atrium. The observation of the UA was performed via the middle site of the free-floating umbilical cord. The UPA was imaged in proximity to the gestational sac.

The pulsed-wave Doppler (PWD) sonography mode of the device was turned on to obtain the waveforms of the relevant vessels. The sample volume was set to $1 \mathrm{~mm}$ and placed in the centre of the vessel. At least three uniform waveforms were recorded to minimize variations due to observational factors. Colour flow mapping and PDW sonography were conducted every 3 days from Day 21 to 60 of pregnancy while the queens were positioned in dorsal recumbency. The angle of insonation was approximately $60^{\circ}$ during spectral measurements. Pulsatility and resistance index (PI and RI) of FA, FVC, UA, and UPA were automatically calculated by the device.
\end{abstract}

Statistical analysis

Statistical analyses were made with Statistical Package for the Social Sciences 10.0 (SPSS). To compare the values between pregnancy days, the General Lineer Model (GLM) was used.

\title{
Results
}

The length of the D-USG per cat was approximately $20 \mathrm{~min}$. Parturitions occurred between Days 61-63 of pregnancy. Therefore, the last Doppler examinations were performed on Day 60 in all queens. All of the parturitions occurred through natural delivery.

The flow of FA was only systolic in the first examination. The end diastolic flow was visualized beginning from Day 27 of pregnancy in all queens. The mean PI values of FA were almost constant throughout pregnancy. Although a declining trend was seen in the mean RI of FA, a comparison with earlier sampling periods revealed that significant decreases $(P<0.05)$ did not occur until Day 30 and 60. The time-dependent changes in PI and RI of FA are presented in Fig. 1.

The FVC was easily detected nearly parallel to the aorta and visualized with a triphasic waveform. The mean PI and RI values of FVC are presented in Fig. 2.

Waveforms of UA were only systolic until Day 36. On Day 36, diastolic flow could be detected and waveforms of UA retained their characteristic saw-tooth appearance until birth. The mean PI of UA on Day 21 was 1.58 . PI recorded in the last two examinations (1.31 on Day 57 and 1.29 on Day 60) were significantly lower than all the others $(P<0.05)$. The mean RI of UA was 0.84 on Day 21. Although a gradual decrease was seen after Day 36 of pregnancy, the mean values of the last three examinations $(0.77,0.75$, and 0.74 , respectively) were significantly lower $(P<0.05)$ than the first ten (Fig. 3$)$.

The UPA was easily visualized in proximity to the gestational sac. The time-dependent changes in PI and RI of UPA are presented in Fig. 4. 

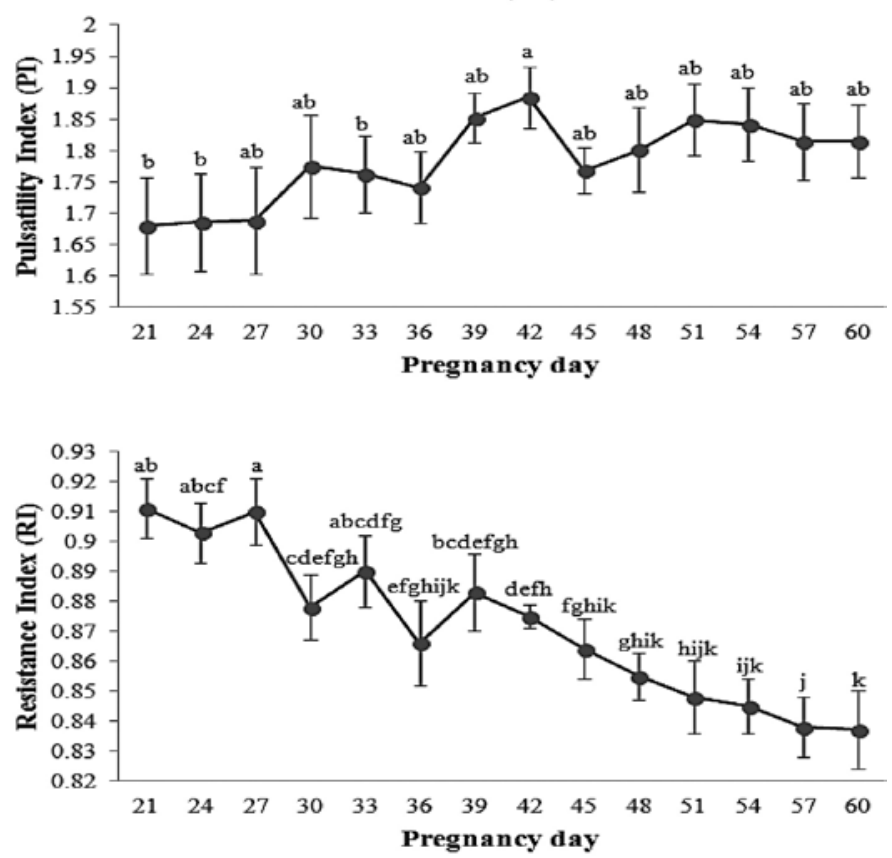

Fig. 1. Time-dependent changes in Pulsatility index (PI) and Resistance index (RI) of fetal aorta a,b,c,d,e,f,g,h,i,j,k: Values not sharing the same superscript are significantly different $(P<0.05)$
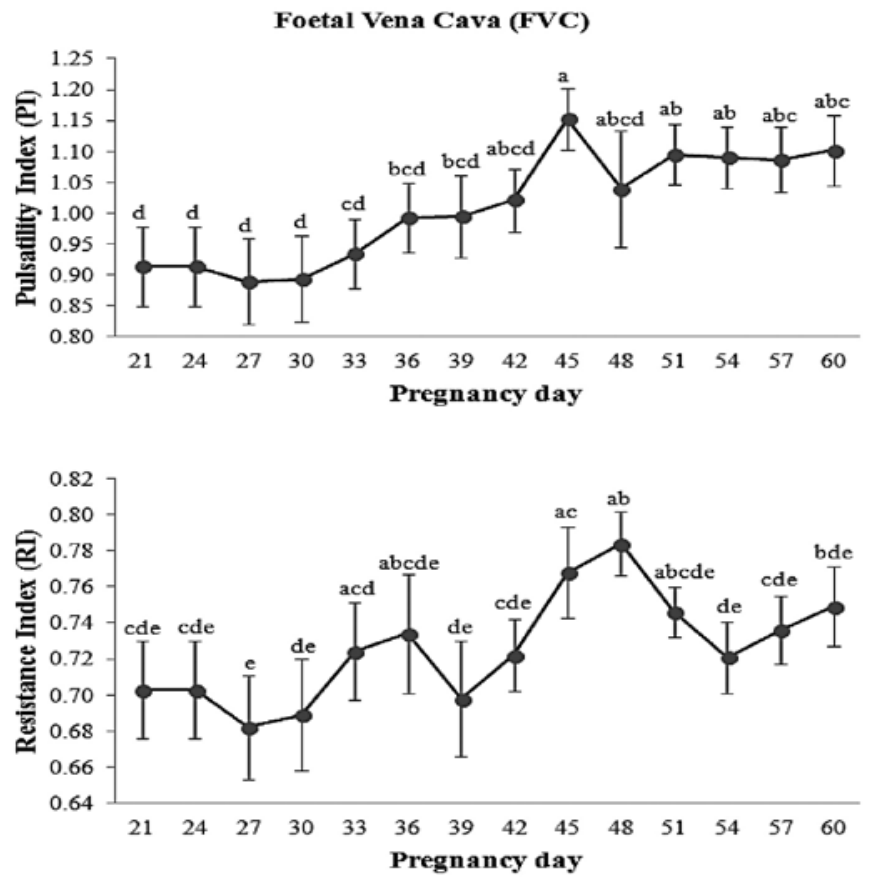

Fig. 2. Time-dependent changes in Pulsatility index (PI) and Resistance index (RI) of fetal vena cava a,b,c,d,e: Values not sharing the same superscript are significantly different $(P<0.05)$ 

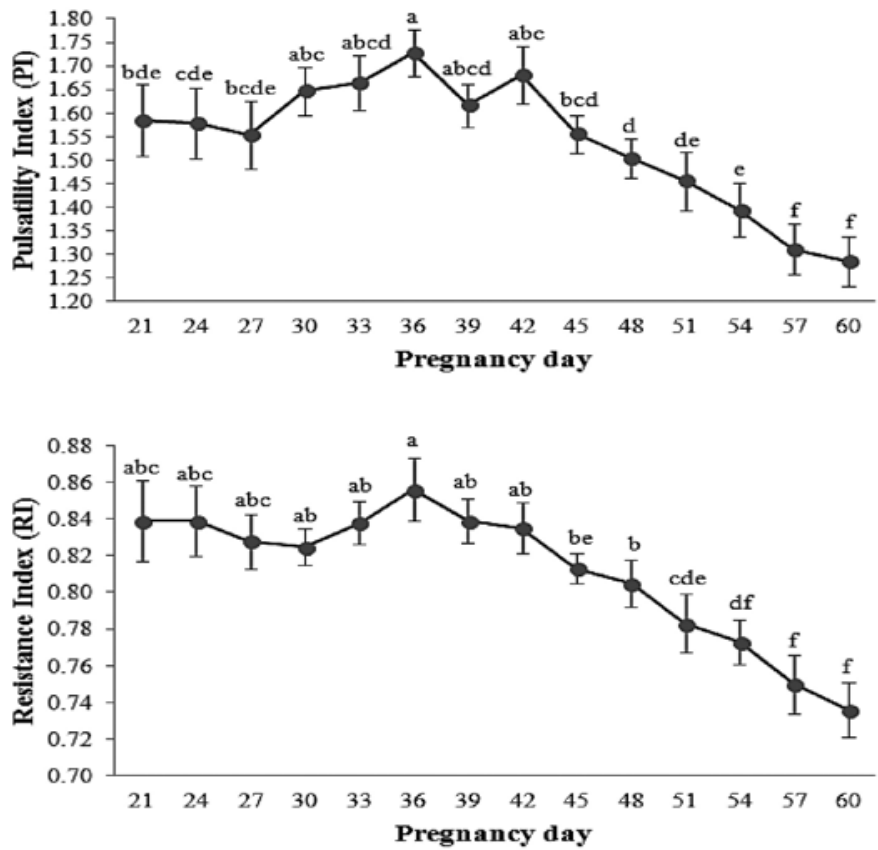

Fig. 3. Time-dependent changes in Pulsatility index (PI) and Resistance index (RI) of umbilical artery a,b,c,d,e,f: Values not sharing the same superscript are significantly different $(P<0.05)$
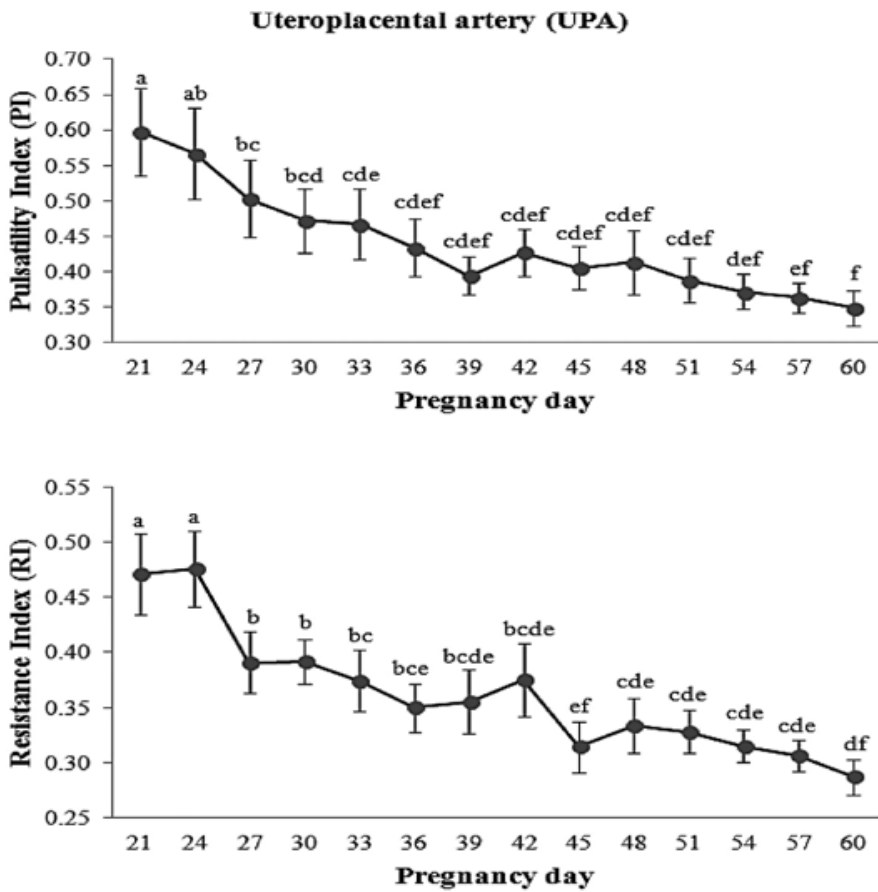

Fig. 4. Time-dependent changes in Pulsatility index (PI) and Resistance index(RI) of uteroplacental artery a,b,c,d,e,f: Values not sharing the same superscript are significantly different $(P<0.05)$ 


\section{Discussion}

The alteration in the waveform of FA was consistent with Scotti et al. (2008) and Pereira et al. (2012). Despite the minimal use of foetal aorta Doppler findings in assessing foetal well-being, research indicates that the disappearance, reversal, or decrease in the end diastolic flow of the foetal aorta may be an indicator of progressive foetal hypoxia (Lees et al. 2003). No abnormality in the waveform of FA was encountered in this study. In accordance with previous reports (Di Salvo et al. 2006; Scotti et al. 2008; Polisca et al. 2010), the presence of the end diastolic flow that we observed in foetal aorta waveforms beginning from Day 27 resulted from the significant decrease in the RI value of the foetal aorta between Days 27 and 30. The declining trend in the subsequent sampling periods in our study may result from the negative correlation between resistance in tissues and vascular perfusion due to vessel enlargement during the progression of pregnancy (Ginther and Matthew 2004; Scotti et al. 2008).

Pereira et al. (2012) reported that both PI and RI values of FVC were relatively stable throughout gestation. In another study conducted by Scotti et al. (2008), PI values of FVC did not decrease significantly until 2-3 weeks before parturition, whereas no significant differences were observed for RI values between consecutive sampling times. When we compared the mean PI and RI values of each sampling time to the previous one, no significant differences were found throughout the gestational period, in line with the previously mentioned results (Scotti et al. 2008; Pereira et al. 2012). Therefore, in our estimation, the qualitative Doppler findings of FVC may not provide reliable information about the gestational age or probable delivery time.

The waveform of FVC is observed to have a triphasic character, with "s," "d," and "a," waves and analyzing this waveform can help assess foetal well-being in human medicine (Shaun and Thomas 2017). It is recommended that foetal venous flow velocities be assessed in foetuses subject to retarded intrauterine growth (Huisman 2001). No reversal or absence of the a-wave in the FVC, and no intrauterine growth retardation, were observed in the foetuses used in this study.

The first day of diastolic flow in UA was similar to that found in previous studies (Scotti et al. 2008; Pereira et al. 2012; Blanco et al. 2014). The absence of the end diastolic flow is an indicator of foetal vascular system problems arising from placental insufficiency in human medicine (Gerber et al. 2006). No absence of umbilical artery end diastolic flow and no placental insufficiency was observed in this study. The declining trend in PI and RI of UA after Day 36 was in accordance with that found in previous studies (Di Salvo et al. 2006; Scotti et al. 2008; Pereira et al. 2012; Blanco et al. 2014), which relate this decline to the progressive development of foetal/placental circulation. Giannico et al. (2015) reported significant declines for umbilical artery RI in pregnant bitches in the prepartum time periods of $72-48,24-12,12-6$, and $6-1 \mathrm{~h}$. The significant reduction in RI of UA after Day 54 compared to the first ten measurements in our study supports Giannico et al.'s (2015) finding that this indicator can be used as a predictor of delivery time. Moreover, the decreased PI values of UA on Day 57 and 60 suggest that also this indicator can be used for determining delivery time in queens. This finding, however, needs to be supported by further studies that investigate the unidentified underlying mechanisms causing alterations in these indices prior to labor.

Small uteroplacental arteries in feline pregnancy were easily found next to gestational sacs, in parallel with the findings of Nautrup (1998) for canine pregnancy. We found a gradual decline in PI and RI of UPA with advancing pregnancy, in harmony with the results of Di Salvo et al. (2006) and Scotti et al. (2008). These values were significantly decreased on Day 60 compared to the first five measurement times for PI and to the first six measurement times for RI. The decrease in the indices of UPA throughout pregnancy is associated with placental development and with histological changes of the spiral arteries 
as pregnancy progresses (Aardema et al. 2001). The non-significance of the changes in the indices of UPA compared to previous measurement times, especially during the last period of pregnancy, calls into question the usability of these indices as a predictor of delivery time. These indices, however, may vary due to placental oedema prior to delivery. This possible correlation should be clarified via more frequent measurement times much closer to delivery time.

Doppler ultrasonography is a noninvasive and practicable procedure in pregnant queens. The findings of this study represented progressive changes in D-USG PI and RI values as well as changes in characteristic blood-flow waveforms of foetal and foeto-maternal vessels throughout pregnancy. The Doppler findings for FA and FVC do not appear to predict delivery time, whereas the decreased PI and RI values of UA may be used as precursors for delivery time in queens. The use of FA and FVC in the evaluation of foetal well-being in human medicine should also be investigated in cats. Just as FA and FVC are used to evaluate foetal well-being in human pregnancies, changes of these vessels should be studied in queens with impaired pregnancies. The usefulness of UPA for determining delivery time requires further research, including more frequent measurement times much closer to delivery time. Furthermore, the reference of D-USG indices for each vessel should be reassessed by studies involving larger numbers of animals. There is also a need for comparative studies involving impaired feline pregnancies.

\section{Acknowledgements}

This research was presented in V. National Congress of Turkish Society of Veterinary Gynaecology, with the support of the Research Fund of Istanbul University (BAP) (Grant number 37251). The authors are also grateful to Prof. Dr. Bülent Ekiz for statistical analysis and Esra Çalişkan Karaçam, DVM for the assistance of Doppler ultrasonography examinations.

\section{Conflict of Interest}

The authors declare that they have no conflict of interest.

\section{References}

Aardema MW, Oosterhof H, Timmer A, Van Rooy I, Aarnoudse IG 2001: Uterine artery Doppler flow and uteroplacental vascular pathology in normal pregnancies and pregnancies complicated by pre-eclampsia and small for gestational age fetuses. Placenta 22: 405-411

Blanco PG, Rodriguez R, Olguin S, Rube A, Tortora M, Gobello C 2014: Doppler ultrasonographic assesment of maternal and fetal arteries during normal feline gestation. Anim Reprod Sci, 146: 63-69

Blanco PG, Vercellini R, Rube A, Rodriguez R, Arias DO, Gobello C 2016: Evaluation of feline uterine and umbilical arteries blood flow in a pharmacologically induced abnormal gestation model. Theriogenology $\mathbf{8 6}$ : 2323-2327

Di Salvo P, Bocci F, Zelli R, Polisca A 2006: Doppler evaluation of maternal and fetal vessels during normal gestation in the bitch. Res Vet Sci 81: 382-388

Elmetwally M, Rohn K, Meinecke-Tillmann S 2016: Noninvasive color Doppler sonography of uterine blood flow throughout pregnancy in sheep and goats. Theriogenology 85: 1070-1079

Erdogan G 2018: Using of Doppler ultrasonography in veterinary gynecology. J Vet Sci Obstet Gynecol-Special Topics 4: 43-49

Ferreira JC, Canesin HS, Ignacio FS, Rocha NS, Pinto CR, Meira C 2015: Effect of age and endometrial degenerative changes on uterine blood flow during early gestation in mares. Theriogenology 84: 1123-1130

Filkins K, Koos JB 2005: Ultrasound and fetal diagnosis. Curr Opin Obstet Gynecol 2005 17: 183-191

Fleischer AC, Goldstein RB, Bruner JP 1994: Doppler sonography in obstetric and gynecology. In: Callen PW (Ed.): Ultrasonography in Obstetrics and Gynecology. WB Saunders Company, Philadelphia, pp. 503-523

Gerber S, Hohlfeld P, Viquerat F, Tolsa JF, Vial Y 2006: Intrauterine growth restriction and absent or reverse enddiastolic blood flow in umbilical artery (Doppler class II or III): A retrospective study of short- and long-term fetal morbidity and mortality. Eur J Obstet Gynecol Reprod Biol 126: 20-26

Giannico AT, Gil EMU, Garcia DAA, Froes TR 2015: The use of Doppler evaluation of the canine umbilical artery in prediction of delivery time and fetal distress. Animal Reproduction Science 154: 105-112

Ginther OJ, Matthew D 2004: Doppler ultrasound in equine reproduction: principles, techniques, and potential. J Equine Vet Sci 24: 516-526 
Huisman TWA 2001: Doppler assessment of the fetal venous system. Semin Perinatol 25: 21-31

Kelley DE, Galvao KN, Mortensen CJ, Risco CA, Ealy AD 2017: Using Doppler ultrasonography on day 34 of pregnancy to predict pregnancy loss in lactating dairy cattle. J Dairy Sci 100: 3266-3271

Lees C, Deane C, Albaiges G 2003: Integrating uterine and fetal Doppler into obstetrics. In: Lees C, Deane C, Albaiges G (Eds): Making Sense of Obstetric Doppler Ultrasound A Hands-on Guide. Arnold, London, pp. 53-59

Maulik D, Mundy D, Heitmann E, Maulik D 2011: Umbilical artery Doppler in the assessment of fetal growth restriction. Clin Perinatol 38: 65-82

Nautrup CP 1998: Doppler ultrasonography of canine maternal and fetal arteries during normal gestation. J Reprod Fertil 112: 301-314

Ouset JC, Kölling M, Newton R, Wright M, Allen WR 2012: Uterine haemodynamics in young and aged pregnant mares measured using Doppler ultrasonography. Equine Vet J Suppl 41: 15-21

Pereira BS, Pinto JN, Freire LMP, Campello CC, Domingues SFS, da Silva LDM 2012: Study of the development of uteroplacental and fetal feline circulation by triplex Doppler. Theriogenology 77: 989-997

Polisca A, Scotti L, Orlandi R, Brecchia C, Boiti C 2010: Doppler evaluation of maternal and fetal vessels during normal gestation in rabbits. Theriogenology $73: 358-366$

Scotti L, Di Salvo P, Bocci F, Pieramati C, Polisca A 2008: Doppler evaluation of maternal and foetal vessels during normal gestation in queen. Theriogenology 69: 1111-1119

Serin G, Gökdal O, Tarimcilar T, Atay O 2010: Umbilical artery Doppler sonography in Saanen goat fetuses during singleton and multiple pregnancies. Theriogenology 74: 1082-1087

Shaun SH, Thomas RE 2017: Antenatal fetal wellbeing. Obstet Gynaecol Reprod Med 27: 44-49

Turna Yilmaz O, Gündüz MC, Evkuran Dal G, Uçmak M, Günay Uçmak Z, Karaçam E, Kaşıkçı G, Kılıçarslan MR 2017: Evaluation of changes in Doppler ultrasonography indices and levels of maternal serum angiogenic factors throughout pregnancy in ewes. Theriogenology 89: 183-191

Turna Yılmaz O, Uçmak M, Günay Z, Çalışkan Karaçam E, Erzengin ÖM 2014: Doppler Evaluation of Fetal and Feto-Maternal Vessels During Dystocia in Cats: Four cases. Kafkas Veteriner Fakültesi Dergisi 20: 971-974 